\title{
Energy Decomposition Analysis for Metal Surface - Adsorbate Interactions by Block Localized Wave Functions
}

Ruben Staub, ${ }^{\dagger}$ Marcella lannuzzi, ${ }^{\ddagger}$ Rustam Z. Khaliullin, $₫$ and Stephan N.

$$
\text { Steinmann } n^{* \dagger}
$$

$\dagger$ †niv Lyon, Ecole Normale Supérieure de Lyon, CNRS Université Lyon 1, Laboratoire de

Chimie UMR 5182, 46 allée d'Italie, F-69364, LYON, France

$\ddagger$ Institut für Chemie, University of Zurich, Winterthurerstrasse 190, CH-8057 Zurich,

Switzerland

【Department of Chemistry, McGill University, 801 Sherbrooke St. West, Montreal, Québec H3A 0B8, Canada

E-mail: stephan.steinmann@ens-lyon.fr

\begin{abstract}
The energy decomposition analysis based on block localized wave functions (BLWEDA) allows to gain physical insight into the nature of chemical bonding, decomposing the interaction energy in (1) a "frozen" term, accounting for the attraction due to electrostatic and dispersion interactions, modulated by Pauli repulsion, (2) the variationally assessed polarization energy and (3) the charge-transfer. This method has so far been applied to gas- and condensed-phase molecular systems. However, its standard version is not compatible with fractionally occupied orbitals (i.e. electronic smearing) and, as a consequence, cannot be applied to metallic surfaces. In this work, we propose a
\end{abstract}


simple and practical extension of BLW-EDA to fractionally occupied orbitals, termed Ensemble BLW-EDA. As illustrative examples, we have applied the developed method to analyze the nature of the interaction of various adsorbates on $\mathrm{Pt}(111)$, ranging from physisorbed water to strongly chemisorbed ethylene. Our results show that polarization and charge-transfer both contribute significantly at the adsorption minimum for all studied systems. The energy decomposition analysis provides details with respect to competing adsorption sites (e.g., CO on atop, vs. hollow sites) and elucidates the respective importance of polarization and charge transfer for the increased adsorption energy of $\mathrm{H}_{2} \mathrm{~S}$ compared to $\mathrm{H}_{2} \mathrm{O}$. Our development will enable a deeper understanding of the impact of charge transfer on catalytic processes in general.

\section{Introduction}

Analyzing the chemical bond in order to understand the driving force and diversity of bonding is almost as old as quantum mechanics. ${ }^{112}$ Bader's quantum theory of the atom in a molecule ${ }^{3}$ and the natural bond orbitals by Weinhold, $\stackrel{4}{4}$ are among the most popular approaches, but since the decomposition of the chemical bond energy into different contributions is not unique, 5 a multitude of other energy decomposition analysis (EDA) schemes have been developed. ${ }^{6}$ All these different schemes come with their advantages and weaknesses, so that application of several tools can either bring contradicting results or provide confidence that at least the trend is captured accurately. ${ }^{7}$ Most of these tools are developed having molecules in mind and their application to the bonding between surfaces and adsorbates is comparatively rare. ${ }^{811}$ As one of the few examples, Tonner and co-workers have demonstrated that EDAs on semi-conducting surfaces can provide a deep understanding of coverage effects ${ }^{12}$ and elucidate peculiar bonding mechanisms. $\frac{13}{13}$ Our goal is to extend the EDA based on the block-localized wave function $(B L W)^{14 \mid 15}$ to metallic surfaces. The BLW is designed to localize the electrons in Hilbert space in the Mulliken sense, i.e., in terms of atom-centered basis functions. A typical choice is to restrict the expansion to basis functions of atoms of a given molecule and 
thus excluding contributions from all other basis functions in the system, thus defining one block per molecule. The BLW - also known in this context as absolutely localized molecular orbitals (ALMO) - is variationally optimized and the different block polarize each other. The main advantage of the BLW-EDA compared to other EDAs is that the polarization of fragments in their mutual presence is computed fully variationaly. This allows to rigorously separate the polarization energy from charge-transfer. The charge-transfer in BLW-EDA includes bonding orbital interactions between the fragments, which distinguishes it from the charge-transfer obtained by constrained density functional theory $(\mathrm{CDFT})^{16117}$, where the charge-transfer is defined in real-space, rather than Hilbert space.18

The formulation of the theoretical framework exploited for optimizing the BLW goes back to the works of Stoll and co-workers, $\frac{19}{19}$ who aimed at reducing the computational cost of the self-consistent field (SCF) procedure by using ALMOs. The variational optimization of blocks that are localized in Hilbert space has found other applications over the years, exploiting other properties of the BLW unrelated to computational speed-ups. For instance, BLW has been proposed to be used to remove the basis set superposition error (BSSE) 2021 and BLWEDA has been applied to molecular complexes at the $\mathrm{DFT}^{222}$ or correlated wave function level of theory, ${ }^{25126}$ quantifying hyperconjugation, ${ }^{2728}$ strain energies ${ }^{29}$ and even covalent bonds. ${ }^{30131}$ Futhermore, ALMOs can provide transferable molecular orbitals (where they are also called ELMOs for extremely localized molecular orbitals ${ }^{32}[35$ or fragment densities to be used in X-ray structure elucidations. $\frac{36}{36}$ Through its variational character, BLW also provides the unique opportunity to directly assess the impact of electron delocalization on the properties of molecules by comparing computed NMR chemical shifts ${ }^{37 / 38}$ and J-couplings ${ }^{39}$ for (de-)localized states, which can be seen as the comparison between a single Lewis structure and the true, electron delocalized state. Similarly, the electronic structure of the electron localized state can be analyzed by scalar fields such as the electron localization function (ELF) or the localized orbital locator (LOL) in order to shed more light on the consequences of electron delocalization on the electronic structure. ${ }^{40 \mid 41}$ Due to the variational definition of the 
polarization energy, other applications involve investigating of the impact of polarization on the hydration shell of ionic solutes ${ }^{42}$ and comparison between the BLW polarization energy and polarizable force fields, which has also been exploited to parametrize first principles based force fields. $\frac{43144}{\underline{4}}$

Heterogeneous catalytic reactions involving metal surfaces or particles are involved in many major industrial processes, such as selective hydrogeneations in refineries, ammonia synthesis, steam reforming etc. Furthermore, metal catalysts are key in heterogeneous electrocatalysis, which is promising to improve the efficiency of electrolysis, fuel cells, $\mathrm{CO}_{2}$ reduction, but also the synthesis of fine chemicals. $\frac{45}{5}$ Therefore, analysis of the interaction of adsorbates with metallic surfaces can provide valuable insights for the design of novel, more efficient catalysts. Furthermore, when considering metal alloys, the difference between electronic and ensemble effects is a widely discussed topic. ${ }^{46}$ BLW would allow to construct an electron localized (neutral) state of a given secondary element, clearly resolving the electronic (charge-transfer between the two metals) and ensemble effect.

The formulation of BLW is based on the assumption of doubly occupied orbitals, although extensions to spin-unrestricted systems exist, $\stackrel{47148}{ }$ where the system is assumed to have a gap between the highest occupied molecular orbital (HOMO) and the lowest unoccupied molecular orbital (LUMO). Metallic electronic structures are, instead, characterized by a continuum of energy levels over the Fermi energy, leading to partially occupied states.

In this work, we propose an extension of the BLW approach based on a mean field approximation, to which we refer as Ensemble BLW-EDA. In the following, we first present the conventional SCF procedure for metallic systems, followed by the main notions of BLW. Then, we combine the two, extending BLW to metallic systems. Finally, we provide computational details and applications to the prototypical adsorption of molecules $\left(\mathrm{H}_{2} \mathrm{O}\right.$, $\mathrm{H}_{2} \mathrm{~S}, \mathrm{C}_{2} \mathrm{H}_{4}$ and $\left.\mathrm{CO}\right)$ on $\mathrm{Pt}(111)$. 


\section{Methodology}

\subsection{Ensemble DFT}

The ground-state of a metallic system, i.e., where the density of states around the Fermi level is continuous, cannot be described by a single quantum state. Rather, an ensemble of states is required, both for a physically sound description and for a smooth convergence of the wave function optimization in a self-consistent field (SCF) process. The different quantum states within the ensemble are weighted according to their probability in order to determine observables. Let $\hat{A}$ be an operator, and $\Omega$ is the set of all possible quantum states of a given system. Then, the expectation value $\langle A\rangle_{\Omega}$ of the ensemble is:

$$
\langle A\rangle_{\Omega}=\sum_{\chi \in \Omega}\left\langle\Psi_{\chi}|\hat{A}| \Psi_{\chi}\right\rangle p_{\chi}
$$

where $\Psi_{\chi}$ is the wavefunction associated with the quantum state $\chi . \Psi_{\chi}$ spans the groundand single excited state determinants of the system. $p_{\chi}$ is the probability that the real system is in the quantum state $\chi$, based on the energy of $\chi$.

In the present context, we are interested in the reformulation of the 1-electron density operator $\hat{\rho}$. In the Hartree-Fock approximation, and in the case of orthonormal molecular orbitals $\psi_{i}, \hat{\rho}$ can be written as:

$$
\hat{\rho}=\sum_{\text {occ } \psi_{i} \in \Psi}\left|\psi_{i}\right\rangle\left\langle\psi_{i}\right|
$$

Dealing explicitly with ensembles is computationally inefficient and would require dedicated implementations. Since singly excited determinants do not overlap with the ground-state determinant, the same set of molecular orbitals can be used to construct all the relevant

quantum states of the system. Therefore, the ensemble 1-electron density $\left(\langle\hat{\rho}\rangle_{\Omega}\right)$ can be written in a convenient manner by considering the population of the orbitals (also known as occupation numbers, $n_{i}$ ) instead of the probability of quantum states: 


$$
\begin{aligned}
\langle\hat{\rho}\rangle_{\Omega} & =\sum_{\chi \in \Omega} p_{\chi} \hat{\rho}_{\chi}=\sum_{\chi \in \Omega} p_{\chi} \sum_{\psi_{i} \in \Psi_{\chi}}\left|\psi_{i}\right\rangle\left\langle\psi_{i}\right|= \\
& =\sum_{i}\left|\psi_{i}\right\rangle\left\langle\psi_{i}\left|n_{i}=\sum_{i}\right| \sqrt{n_{i}} \psi_{i}\right\rangle\left\langle\sqrt{n_{i}} \psi_{i}\left|=\sum_{i}\right| \psi_{i}^{\prime}\right\rangle\left\langle\psi_{i}^{\prime}\right|
\end{aligned}
$$

where $i$ is the sum over all molecular orbitals, i.e., solutions of the Fock equation. In Eq. 4 we have introduced the rescaled orbitals:

$$
\left|\psi_{i}^{\prime}\right\rangle=\sqrt{n_{i}}\left|\psi_{i}\right\rangle
$$

which are particularly convenient computationally. The occupation number $n_{i}$ is related to the probability for the orbital $\psi_{i}$ to be occupied. For finite temperatures, they are determined based on the energy $\epsilon_{i}$ of $\psi_{i}$, invoking Fermi-Dirac statistics:

$$
n_{i}=\frac{1}{\exp \left(\frac{\epsilon_{i}-\mu}{k T}\right)+1}
$$

where $\mu$ is the Fermi level, $k$ is Boltzmann's constant, and $\mathrm{T}$ is the (electronic) temperature. With these rescaled orbitals $\left|\psi_{i}^{\prime}\right\rangle$, the mixed-state 1-electron density $R_{\Omega}$ can be easily computed.

$$
R_{\Omega}=\mathrm{CNC}^{\dagger}
$$

where $\mathrm{C}$ is the molecular coefficient matrix and $\mathrm{N}$ is a diagonal matrix of Fermi weights $n_{i}$ according to Eq. 66. Yang and co-workers have generalized this reformulation to any method that can be reformulated based on the non-interacting Greens function, allowing to apply the orbital scaling to virtual orbitals and thus to compute correlation energies with MP2 or RPA for systems with fractional electrons. ${ }^{49}$ With a mild approximation, these scaled orbitals can also be used for coupled cluster computations for systems with fractional electrons. ${ }^{50}$ 


\subsection{BLW formalism}

The basic idea of the BLW formalism is to express the wave function of the system in terms of blocks of localized orbitals. In the present context, the orbitals are always localized on a subset of atoms, so that the BLW partitions the atom-centered basis functions in mutually exclusive blocks and imposes thereby a localization of the wave function in Hilbert space.

Formally, a block $B_{m}$ is defined as a set of $N_{m}$ basis functions $\left\{\phi_{1}^{m} \ldots \phi_{N_{m}}^{m}\right\}$, such that each basis function is associated with exactly one block, i.e. $\sum_{m} N_{m}=N$, where $N$ is the total number of basis functions of the system. In this work, the set of basis functions $\left\{\phi_{1}^{m} \ldots \phi_{N_{m}}^{m}\right\}$ associated with the block $B_{m}$ is the union of all $N_{j}$ basis set functions $\left\{\phi_{1}^{j} \ldots \phi_{N_{j}}^{j}\right\}$ used to describe the $j_{m}$ atoms of $B_{m}$, so that $\sum_{m} j_{m}$ equals to the total number of atoms in the system. Similarly, the electrons of the system are assigned to a given block. In our case, all blocks are neutral.

An ALMO is defined as a linear combination of basis functions associated with the same block. Therefore, an orbital $\psi_{i^{m}}$ pertaining to block $B_{m}$ is written:

$$
\left|\psi_{i^{m}}\right\rangle=\sum_{\nu} C_{\nu, i}^{m}\left|\phi_{\nu}^{m}\right\rangle
$$

where $\mathbf{C}$ is the orbital coefficient matrix for the entire system, while $\mathbf{C}^{\mathbf{m}}$ is the matrix of block $B_{m}$. We have used greek letters to label atomic orbital basis functions and $i^{m}$ indexes ALMOs of a given block $B_{m}$.

The global orbital coefficient matrix $\mathbf{C}$, which spans all molecular orbitals and, thus, has dimensions of $N \times N$, has a block-diagonal structure:

$$
\mathbf{C}=\left(\begin{array}{cccc}
\mathbf{C}^{\mathbf{1}} & 0 & \cdots & 0 \\
0 & \mathbf{C}^{\mathbf{m}} & \cdots & 0 \\
\vdots & \vdots & \ddots & \vdots \\
0 & 0 & \cdots & \mathbf{C}^{\sum_{\mathbf{m}}}
\end{array}\right)
$$


where $\mathbf{C}^{\mathbf{m}}$ is the orbital coefficient matrix restricted to the block $B_{m}$. The orbital coefficients $C_{\nu, i}^{m}$ are variationally optimized according to the local diagonalisation-based SCF algorithm developed by Stoll 19 and implemented in CP2K as the first stage of the two-step "ALMO-SCF" scheme. .51

In general, locality and orthogonality constraints cannot be both satisfied simultaneously. $\underline{52}$ Therefore, ALMOs are inherently non-orthogonal between blocks, although they can be kept orthonormal within a block without loss of generality. In this study, we will work under this assumption. Hence, the ALMO overlap matrix $(\sigma)$ has identity-like diagonal blocks, but non-zero entries for overlaps between blocks.

In practice, the so called reciprocal (or bi-orthogonal) occupied orbitals $\left|\tilde{\psi_{i^{m}}}\right\rangle$ are defined such that $\left\langle\tilde{i^{m}} \mid \psi_{j^{l}}\right\rangle=\delta_{i^{m} j^{l}}$ :

$$
\left|\tilde{i_{i^{m}}}\right\rangle=\sum_{l, j}\left|\psi_{j^{l}}\right\rangle \sigma_{i^{m} j^{l}}^{-1}, \quad \tilde{\mathbf{T}}=\mathbf{T} \sigma^{-1}
$$

where $l$ goes over all blocks and $j^{l}$ indexes the occupied orbitals of block $B_{l}, \tilde{\mathbf{T}}$ is the coefficient matrix of reciprocal occupied orbitals and $\mathbf{T}$ is the occupied part of Eq. 9. To be explicit, $\left|\tilde{i_{i^{m}}}\right\rangle$ is expanded in terms of all basis functions of the system and not only of block $B_{m}$.

Reciprocal orbitals enable one to rewrite the Fock equations. As Stoll has demonstrated, the self-consistent solution of projected eigenvalue equations for each block is equivalent to finding variationally optimal ALMOs.

$$
\begin{gathered}
\hat{F}^{m}\left|\psi_{i}^{m}\right\rangle=\epsilon_{i}^{m}\left|\psi_{i}^{m}\right\rangle, \quad \hat{F}^{m}=\left(\hat{\mathbb{1}}-\hat{\rho}+\hat{\rho}^{m \dagger}\right) \hat{F}\left(\hat{\mathbb{1}}-\hat{\rho}+\hat{\rho}^{m}\right) \\
\hat{\rho}^{m}=\sum_{i \in B_{m}}\left|\tilde{\psi}_{i^{m}}\right\rangle\left\langle\psi_{i^{m}}\left|=\sum_{l, \xi, \nu} R_{\xi \nu}^{m}\right| \phi_{\xi}^{l}\right\rangle\left\langle\phi_{\nu}^{m}\right|
\end{gathered}
$$

where $\mathbb{1}$ is the identity matrix, $\hat{F}$ is the conventional Fock operator, $\hat{F}^{m}$ is the Fock operator projected on block $B_{m}, \hat{\rho}$ is the density operator of the entire system, $\hat{\rho}^{m}$ is the non-Hermitian operator that represents the density of the block defined by using only ALMOs of block $B_{m}$, 
and $R^{m}$ is the associated block density matrix. $\nu$ is a basis function of block $B_{m}$ (see Eq. 8), while $\xi$ indexes the atom centered basis function of blocks $B_{l}$.

At each SCF iteration, these projected Fock equations are solved independently for each block, but coupled to each other between successive iterations due to the projection operators. In other words, each block $B_{m}$ is optimized in the environment generated by all other blocks.

Since in mixed-state theory it is common to deal with orthonormal orbitals, we briefly discuss the connection between the use of reciprocal orbitals and a Lowdin orthonormalization:

$$
\left.{ }^{L} \psi_{i}\right\rangle=\sum_{\text {occ } j}\left|\psi_{j}\right\rangle \sigma_{i j}^{-\frac{1}{2}}, \quad{ }^{\mathbf{L}} \mathbf{T}=\mathbf{T} \sigma^{-\frac{1}{2}}
$$

where $i$ and $j$ are general indexes, $\sigma^{-\frac{1}{2}}$ is the square root inverse of the overlap matrix and ${ }^{\mathbf{L}} \mathbf{T}$ is the Lowdin orbital coefficient matrix. The 1-electron density is equivalently expressed through the Lowdin orthonormalized molecular orbitals and the use of reciprocal orbtials, the latter avoiding the expensive computation of $\sigma^{-\frac{1}{2}}$ :

$$
\begin{gathered}
\left.\hat{\rho}=\sum_{\text {occ } i}\left|{ }_{i}^{L} \psi_{i}\right\rangle{ }^{L} \psi_{i}\left|=\sum_{\text {occ } i}\right| \tilde{\psi}_{i}\right\rangle\left\langle\psi_{i}\left|=\sum_{\text {occ } i} \sum_{\nu, \xi} \tilde{T}_{\nu i}\right| \phi_{\nu}\right\rangle\left\langle\phi_{\xi}\left|T_{\xi i}=\sum_{\nu, \xi} \sum_{\text {occ } i} T_{\xi i} \tilde{T}_{i \nu}^{\dagger}\right| \phi_{\nu}\right\rangle\left\langle\phi_{\xi}\left|=\sum_{\nu, \xi} R_{\nu \xi}\right| \phi_{\nu}\right\rangle\left\langle\phi_{\xi}\right| \\
\mathbf{R}={ }^{\mathbf{L}} \mathbf{T}^{\mathbf{L}} \mathbf{T}^{\dagger}=\mathbf{T} \tilde{\mathbf{T}}^{\dagger}=\mathbf{T}\left(\mathbf{T} \sigma^{-1}\right)^{\dagger}=\mathbf{T} \sigma^{-1} \mathbf{T}^{\dagger}
\end{gathered}
$$

where $\mathbf{R}$ is the conventional 1-electron density matrix. $\mathbf{R}$ can then be fed to standard routines to determine the electron density in real space, compute gradients and so forth.

\subsection{Ensemble BLW}

\subsubsection{Exact Ensemble Formulation}

In order to adapt the BLW formalism to an ensemble formulation, we need to adapt the density matrices $\mathbf{R}$ or, equivalently, the construction of the reciprocal orbitals (Eq. 10). In other words, we apply the general formula (Eq. 3) for the computation of an ensemble density 
matrix $\mathbf{R}_{\Omega}$ to non-orthogonal molecular orbitals:

$$
\mathbf{R}_{\Omega}=\sum_{\chi \in \Omega} p_{\chi} \mathbf{T}_{\chi}=\sum_{\chi \in \Omega} p_{\chi} \mathbf{T}_{\chi} \sigma_{\chi}^{-1} \mathbf{R}_{\chi}^{\dagger}=\sum_{\chi \in \Omega} p_{\chi} \mathbf{T}_{\chi}\left(\mathbf{T}_{\chi}^{\dagger} \mathbf{S} \mathbf{T}_{\chi}\right)^{-1} \mathbf{T}_{\chi}^{\dagger}
$$

where $p_{\chi}$ is the probability that the real system is in the quantum state $\chi, \mathbf{R}_{\chi}$ is the density matrix of this quantum state, $\mathbf{T}_{\chi}$ is the occupied orbital coefficient matrix associated with the wavefunction $\Psi_{\chi}, \sigma_{\chi}^{-1}$ is the overlap matrix of the quantum state $\chi$, and $\mathbf{S}$ is the basis set function overlap matrix which is common for all quantum states, since it is a property of the geometry and basis set.

Let us consider a general orbital coefficient matrix $\mathbf{C}$ containing all the localized orbitals used to construct every $\mathbf{T}_{\chi}$, the occupied orbital matrix of quantum state $\chi$. Therefore, one can construct any $\mathbf{T}_{\chi}$ from $\mathbf{C}$ :

$$
\mathbf{T}_{\chi}=\mathbf{C} \Delta_{\chi}=\mathbf{C}\left(\begin{array}{cccc}
\delta_{1 \chi} & 0 & \cdots & 0 \\
0 & \delta_{2 \chi} & \cdots & 0 \\
\vdots & \vdots & \ddots & \vdots \\
0 & 0 & \cdots & \delta_{n+k \chi}
\end{array}\right), \quad \delta_{i \chi}=\left\{\begin{array}{cc}
1 & \text { if } \psi_{i} \in \Psi_{\chi} \\
0 & \text { otherwise }
\end{array}\right.
$$

where $\Delta_{\chi}$ can be seen as a rescaling matrix with a dimension of $N \times N$.

Combining Eq. 17 with Eq. 16, we obtain the following reformulation:

$\mathbf{R}=\sum_{\chi \in \Omega} p_{\chi} \mathbf{R}_{\chi}=\sum_{\chi \in \Omega} p_{\chi} \mathbf{T}_{\chi} \sigma_{\chi}^{-1} \mathbf{T}_{\chi}^{\dagger}=\sum_{\chi \in \Omega} p_{\chi} \mathbf{C} \Delta_{\chi} \sigma_{\chi}^{-1} \Delta_{\chi} \mathbf{C}^{\dagger}=\mathbf{C}\left(\sum_{\chi \in \Omega} p_{\chi} \Delta_{\chi} \sigma_{\chi}^{-1} \Delta_{\chi}\right) \mathbf{C}^{\dagger}=\mathbf{C}^{\Omega} \sigma^{-1} \mathbf{C}^{\dagger}$

where ${ }^{\Omega} \sigma^{-1}=\sum_{\chi \in \Omega} p_{\chi} \Delta_{\chi} \sigma_{\chi}^{-1} \Delta_{\chi}$.

Equation 18 requires $\sigma_{\chi}^{-1}$ to be computed for each state involved separately, i.e., it is not an efficient reformulation of Eq. 16. This contrasts with the canonical case, where Eq. 4 provides an efficient reformulation of Eq. 3, since the different quantum states involved are orthonormal among each other. 
If we have $k$ orbitals in addition to the $n$ formally doubly occupied orbitals, then the maximum number $|\Omega|$ of quantum states to evaluate Eq. 17 is:

$$
|\Omega|=\left(\begin{array}{c}
n+k \\
n
\end{array}\right)=\frac{(n+k) !}{n ! k !}
$$

In the worst case, $k=n,|\Omega| \approx \frac{2^{2 n}}{\sqrt{\pi n}}$, i.e., the number of states to be considered is exponential in $n$. Hence, such a method is not applicable to sizable systems (e.g. a little more than $7 \times 10^{201}$ states for the $\mathrm{Pt}(111)$ surface investigated in the later sections).

Why can there not be a simple reformulation of ensemble BLW, just like in the canonical case? Considering Eq. 14, we can understand that ALMOs are treated as if they would be orthonormalized when computing the density matrix. Therefore, scaling them by their occupation number is not an option, since this lack of normalization will simply be offset by the corresponding $\sigma^{-1}$. A second point of interference when aiming at a simplified ensemble description comes from the interdependence of blocks. Let us consider a two block system. According to Eq. 10, the reciprocal orbitals of block 1 depend on the occupied orbitals of all blocks. Hence, in general (i.e., when the orbitals between blocks overlap) varying the occupations in block 2, enters as a varying environment for block 1 (and vice versa). In other words, the different quantum states in block 1 are subjected to different environments, depending on the quantum states of block 2. To put this in mathematical terms, let us consider two quantum states $\chi$ and $\chi^{\prime}$, with associated wave-function $\Psi_{\chi}$ and $\Psi_{\chi^{\prime}}$ and three different orbitals $\psi_{1}, \psi_{2}$ and $\psi_{3}$ (considered doubly occupied) such that $\left\{\psi_{1}, \psi_{2}\right\} \subseteq \Psi_{\chi}$ and $\left\{\psi_{1}, \psi_{3}\right\} \subseteq \Psi_{\chi^{\prime}}$, but $\psi_{3} \notin \Psi_{\chi}$ and $\psi_{2} \notin \Psi_{\chi^{\prime}}$. If we would like to use the same set of ALMOs for describing $\Psi_{\chi}$ and $\Psi_{\chi^{\prime}}$, then we would like $\tilde{\psi}_{1}$ to be equal to $\tilde{\psi}_{1}{ }^{\prime}$ (respectively the reciprocal orbital of $\psi_{1}$ computed in the quantum state $\chi$ and $\chi^{\prime}$ ) so that Eq. 18 can be simplified in full analogy with the situation when using orthonormalized orbitals. However the reciprocal orbital $\tilde{\psi}_{1}$ in the quantum state $\chi$ does not change by replacing the orbital $\psi_{2}$ by $\psi_{3}$, if and only if $\psi_{1}$ do not overlap with neither $\psi_{2}$ nor $\psi_{3}$, and there exists no $\psi_{i} \notin\left\{\psi_{1}, \psi_{2}, \psi_{3}\right\}$ in 
any quantum state $\chi_{j} \in \Omega$ such that both $\psi_{1}$ and $\psi_{2}$ (or $\psi_{3}$ ) overlap with $\psi_{i}$ :

$$
\left|\tilde{\psi}_{1}\right\rangle=\left|\tilde{\psi}_{1}^{\prime}\right\rangle \Leftrightarrow\left\{\begin{array}{l}
\left\langle\psi_{1} \mid \psi_{2}\right\rangle=0 \\
\wedge\left(\forall \chi \in \Omega, \nexists \psi_{i} \in \Psi_{\chi} \backslash\left\{\psi_{1}, \psi_{2}\right\}, \psi_{1} \in \Psi_{\chi} \rightarrow\left(\left\langle\psi_{1} \mid \psi_{i}\right\rangle \neq 0 \wedge\left\langle\psi_{2} \mid \psi_{i}\right\rangle \neq 0\right)\right) \\
\wedge\left(\text { idem for } \psi_{3} \text { instead of } \psi_{2}\right)
\end{array}\right.
$$

Therefore, the occupation-state dependency of the orthonormalized orbitals is always present, except when the added or removed orbitals does not overlap with the rest of the system. Due to this occupation-state dependency of the orthonormalized orbitals, they cannot be used to construct an ensemble density matrix by rescaling them, in contrast to the canonical case.

As a conclusion, since the orbitals are non-orthogonal, when an orbital occupation is modified the whole system has to re-adapt. Note that this conclusion applies to the use of any non-orthogonal orbitals and not only to ALMOs.

\subsubsection{Mean-field approximation to Ensemble BLW}

The condition to formulate a computationally tractable approximation to ensemble BLW is that the contribution of each orbital to the ensemble density can be computed only once per SCF iteration and can then be weighted by the probability that the real system is in a quantum state containing this orbital (or equivalently, the probability that this orbital is occupied in the real system). In other words, we need to devise a scheme in which we have a common overlap matrix $\sigma$ for all quantum states involved, i.e., we generate an average interaction field (over all quantum states) that is applied to every quantum state. Furthermore, we require that the scheme is equivalent to standard ensemble theory for a single block. This implies that Eq. 6 is applied to each block separately (with the block specific chemical potential $\mu^{m}$ ), so that the total number of electrons in each block remains an integer. With

these two requirements, we arrive at a mean-field approximation to the overlap matrix $\left({ }^{\alpha} \sigma\right)$ :

The interaction (i.e., overlap) between $\left|\psi_{i^{m}}\right\rangle$ and $\left|\psi_{j^{l}}\right\rangle$ from any block, is rescaled by 
$\sqrt{n_{i}} \sqrt{n_{j}}$, while the self-overlap remains unmodified. This rescaling provides an approximate overlap matrix ${ }^{\alpha} \sigma$ :

$$
{ }^{\alpha} \sigma_{i j}=\left\{\begin{array}{l}
\left\langle\psi_{i^{m}} \mid \psi_{i^{m}}\right\rangle=1 \\
\left\langle\psi_{i^{m}} \mid \psi_{j^{\prime}}\right\rangle \sqrt{n_{i}} \sqrt{n_{j}}
\end{array}\right.
$$

Hence, the density matrix ${ }^{\alpha} \mathbf{R}_{\Omega}$ can be written:

$$
{ }^{\alpha} \mathbf{R}_{\Omega}=\mathbf{T}^{\prime \alpha} \sigma^{-1} \mathbf{T}^{\prime \dagger}
$$

where $\mathbf{T}^{\prime}$ is the rescaled orbital coefficient matrix, in close analogy to Eq. 5, for the canonical case.

We use $\left.\left.\right|^{s} \psi_{i^{m}}\right\rangle$ to denote the presence of fractionally occupied ALMOs, i.e., the ones that necessitate the use of ${ }^{\alpha} \sigma .\left|{ }^{s} \psi_{i^{m}}\right\rangle$ have rescaled interactions (overlap), except with themselves. Therefore, we call them "selfish orbitals". Unlike the canonical rescaled orbitals, selfish orbitals cannot be considered "shrunk", they just interact less with their environment:

$$
\forall j \neq i \quad\left\langle\left.{ }^{s} \psi_{i^{m}}\right|^{s} \psi_{j^{l}}\right\rangle=\sqrt{n_{i}} \sqrt{n_{j}}\left\langle\psi_{i^{m}} \mid \psi_{j^{l}}\right\rangle ; \operatorname{but}\left\langle\left.{ }^{s} \psi_{i^{m}}\right|^{s} \psi_{i^{m}}\right\rangle=\left\langle\psi_{i^{m}} \mid \psi_{i^{m}}\right\rangle
$$

where $j$ goes over ALMOs of all blocks.

In practice, at each SCF iteration the orbital coefficients $\mathbf{T}$ are scaled according to the (updated) occupation number, yielding $\mathbf{T}^{\prime}$. With these scaled coefficients, the overlap matrix $\sigma$ is computed. Then, the diagonal of $\sigma$ is set to unity to obtain ${ }^{\alpha} \sigma$. The density matrix is computed using Eq. 22 In brief, except for the use of $\mathbf{T}^{\prime}$ and ${ }^{\alpha} \sigma$, the SCF procedure by Stoll is not modified.

Please note that for non-interacting systems (blocks that are far apart), the mean-field approximation reverts back to the canonical answer. Similarly, if the orbital occupations are either 0 or 2 ( $0 \mathrm{~K}$ limit of a system with a non-vanishing gap), our approximation provides the regular BLW result. 
In our implementation in CP2K, we exploit the eigenvalue based optimization by Stoll $15 \mid 19$, which has been implemented in $\mathrm{CP} 2 \mathrm{~K}^{51 / 53}$ and allows a variational optimization of the ensemble BLW. The computational cost of the extension to partially occupied orbitals is negligible thanks to the use of selfish orbitals and the additional storage used is limited to an array containing the orbital energies.

As discussed in the SI, the error introduced by the mean-field approximation turns out to lead to losses of electrons in the Ensemble BLW. For example, for $\mathrm{H}_{2} \mathrm{O}$ up to 0.005 electrons are lost when the molecule is closest to the metal surface. The energetic consequence on the polarization energy of this electron loss can be estimated to be up to $0.5 \mathrm{kcal} \mathrm{mol}^{-1}$ assuming a metallic system with a typical workfunction around $5 \mathrm{eV}$. As a result, the charge-transfer energy, which does not suffer from this electron loss, is slightly overestimated compared to the polarization energy. Given that water, which is the least strongly adsorbed molecule studied herein, has an adsorption energy of $-9.7 \mathrm{kcal} \mathrm{mol}^{-1}$, such an error is deemed acceptable. In all cases no, or a small loss of electrons is encountered, never a gain in electrons. This is can be rationalized in analogy to Hartree-Fock, where the average electron repulsion overestimates the actual electron repulsion. Hence, our mean-field approximation is an upper bound to exact ensemble BLW. Since ensemble DFT formulation is variational with respect to the electronic free energy (i.e., when accounting for the entropy related to the fractionally occupied orbitals), our Ensemble BLW-EDA defines the charge-transfer through a variational principle for the interaction of adsorbates with metallic surfaces.

\section{Computational Details}

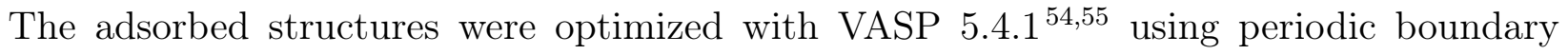
conditions applying the re-optimized Perdew, Burke and Ernzerhofer (PBE) functional to make it compatibles with the non-local van der Waals (vdW) functional, in short optPBE$\mathrm{vdW}^{56}$ functional which is has been found to be, together with PBE-dDsC $\frac{57}{\text {, }}$, an other 
dispersion corrected density functional, most accurate for adsorption energies on $\operatorname{Pt}(111)^{58}$, and is available in CP2K, the code that is used for all Ensemble BLW-EDA computations. An energy cutoff of $400 \mathrm{eV}$ is chosen for the expansion of the plane-wave basis set. The electron-ion interactions are described by the PAW formalism. $\stackrel{5960}{ }$ The $\mathrm{p}(6 \times 6)$ unit cell is built from bulk platinum (2.821 $\AA$ nearest neighbor distance) with four metallic layers. Additional tests regarding the need for K-point sampling reveal that the $\Gamma$-point optimized geometries are very close to the ones obtained with a $3 \times 3 \times 1 \mathrm{~K}$-point mesh. The adsorption energy at the $\Gamma$-point is overestimated by $1-5 \mathrm{kcal} \mathrm{mol}^{-1}$, corresponding to up to $10 \%$ at the equilibrium distance (see Table S2). This accuracy is deemed acceptable for the current purpose, where the relative importance of different interaction energy components and their evolution as a function of the surface - adsorbate distance is analyzed. The out-of-plane vector of the unit cell was chosen to be $\sim 23 \AA$ to achieve a negligible interaction between periodic images.

The molecular orbitals were represented by a double- $\zeta$ Gaussian basis set with one set of polarization functions, called DZVP-MOLOPT-SR-GTH for both BLW-EDA and BSSE corrected SCF DFT simulations. $\frac{61}{6}$ A cutoff of 400 Ry was used to describe the electron density. The exchange-correlation (XC) energy was approximated with the optPBE-vdW 56 functional. The Brillouin zone was described at the $\Gamma$-point. Goedecker, Teter and Hutter (GTH) pseudopotentials $^{62}$ based on the PBE functional were used to describe the interactions between the valence electrons and the ionic cores, and the electronic smearing was approximated by a Fermi-Dirac distribution at $300 \mathrm{~K}$, applied to all computations.

Figure S1 provides a comparison between the total interaction energy $\left(\Delta E_{\text {int }}\right.$, vide infra, Eq. 24) as computed by standard KS-DFT with VASP and CP2K. In both codes, we compare a 10 and a 18 valence electron pseudopotential. The 18 valence electron potential of CP2K gives interaction energies very close to the one provided by VASP, independently if the latter uses 10 or a 18 valence electrons. 


\subsection{Energy Decomposition Analysis}

The newly extended EDA scheme is now applied to charge transfer analysis of adsorption at a metallic surface. We select three prototypical couples of systems (see Figure 11), aiming at describing different types of bonding. The first couple is $\mathrm{H}_{2} \mathrm{O}$ and $\mathrm{H}_{2} \mathrm{~S}$, for which no strong bond formation is expected, although $\mathrm{H}_{2} \mathrm{~S}$ is interacting more strongly with $\mathrm{Pt}(111)$ than water. The second couple compares the di- $\sigma$ and $\pi$ adsorption modes of ethylene, while the third couple investigates the difference between $\mathrm{CO}$ adsorbed on fcc and top sites.

For all these systems, we have computed the interaction energy as a function of the distance between the surface and the adsorbate. All coordinates are fully optimized, except the z-coordinate of the heavy atoms closest to the surface and the two bottom layers that are kept fixed in their bulk position. We define the total interaction energy $\Delta E_{t o t}$ as:

$$
\Delta E_{\text {tot }}=E_{\text {system }}-E_{\text {frag1,opt }}-E_{\text {frag } 2, o p t}-\Delta E_{B S S E}
$$

Where $E_{\text {system }}$ is the standard KS-SCF energy of the full system, $E_{\text {frag,opt }}$ are the corresponding energies of the freely optimized fragments. Since the BLW is only defined in a localized basis set, we have to correct for the basis set superposition error (BSSE), which we do according to the counterpoise procedure of Boys and Bernardi ${ }^{63}$, giving rise to the energy correction $\Delta E_{B S S E}$. Note, however, that BSSE only affects the charge-transfer term, as all other terms are evaluated using exclusively the fragment basis set.

For the energy decomposition analysis, each system is divided into two blocks: a metallic block containing the metal surface, and an adsorbate block containing the adsorbed molecule. As common in BLW-EDA, 2223164 we decompose the total interaction energy $\Delta E_{\text {tot }}$ into the

following terms: preparation or deformation $\left(\Delta E_{\text {deform }}\right)$, the frozen energy term $\left(\Delta E_{\text {frozen }}\right)$ that describes the interaction of the two isolated fragment densities brought together and covers electrostatic interaction and Pauli repulsion ${ }^{[22}$ as well as dispersion interactions ${ }^{64}$, polarization $\left(\Delta E_{p o l}\right)$ energy, obtained by variationally optimizing the BLW, and, finally, the 

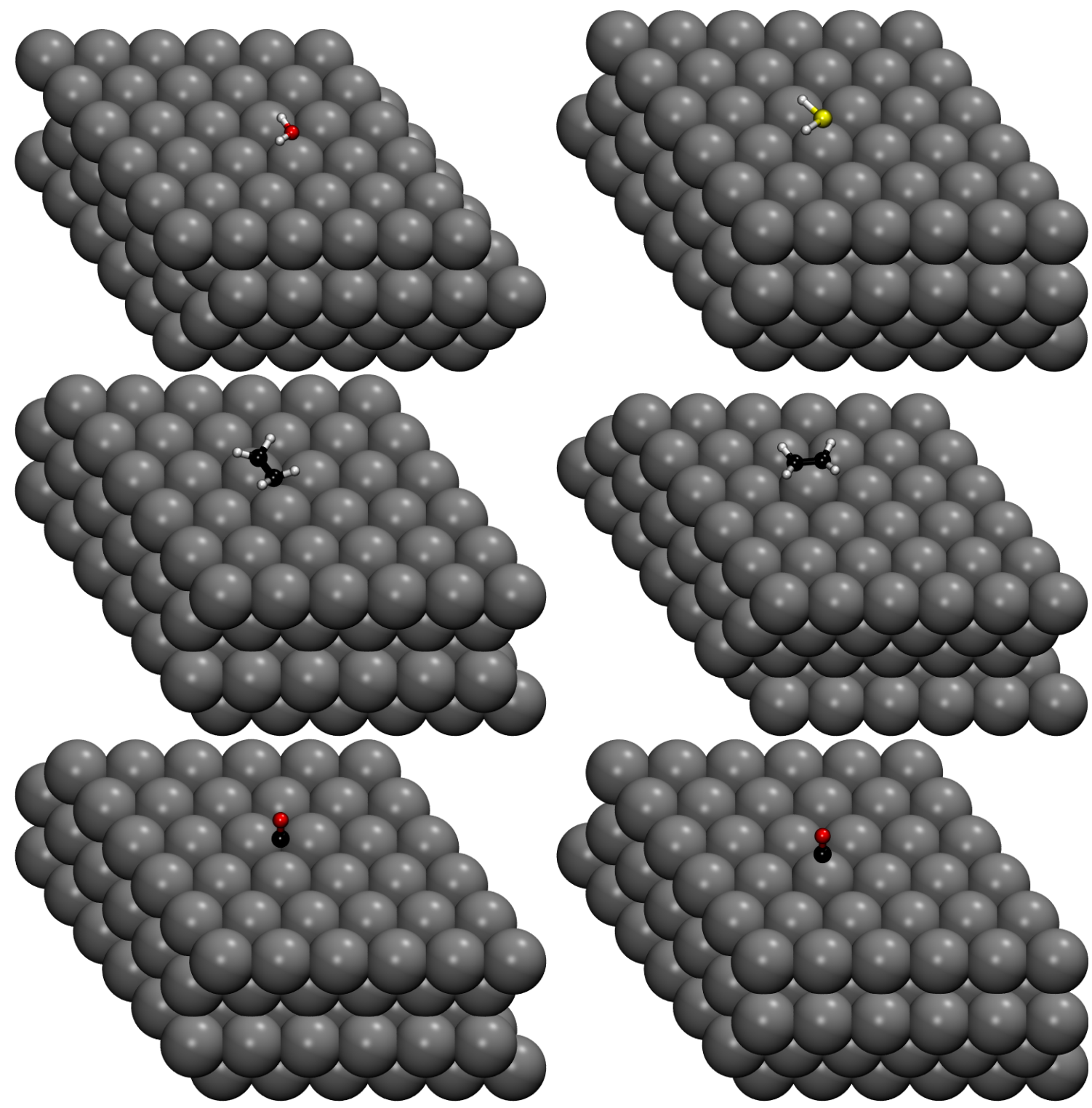

Figure 1: Top: $\mathrm{H}_{2} \mathrm{O}$ (left) and $\mathrm{H}_{2} \mathrm{~S}$ (right) top adsorption on $\mathrm{Pt}(111)$. Middle: Ethylene adsorption in a di- $\sigma$ (left) and $\pi$ adsorption mode. Bottom: CO on fcc (left) and top (right) adsorption sites. All images refer to the equilibrium distance with respect to the surface. 
charge transfer $\left(\Delta E_{C T}\right)$ interaction that includes covalent bond formation.

$$
\Delta E_{\text {tot }}=\Delta E_{\text {deform }}+\Delta E_{\text {frozen }}+\Delta E_{\text {pol }}+\Delta E_{C T}
$$

These terms can be expressed as:

$$
\begin{aligned}
\Delta E_{\text {deform }} & =E_{\text {frag } 1, \text { sys }}-E_{\text {frag } 1, \text { opt }}+E_{\text {frag } 2, \text { sys }}-E_{\text {frag } 2, \text { opt }} \\
\Delta E_{\text {frozen }} & =E_{\text {guess }}-E_{\text {frag } 1, \text { sys }}-E_{\text {frag } 2, \text { sys }} \\
\Delta E_{\text {pol }} & =E_{B L W}-E_{\text {guess }} \\
\Delta E_{C T} & =E_{\text {system }}-E_{B L W}-\Delta E_{B S S E}
\end{aligned}
$$

where $E_{\text {frag,sys }}$ corresponds to the energy of a fragment in its final geometry adopted in the presence of the other fragment. $E_{\text {guess }}$ is the systems energy obtained by a superposition of the fragment density matrices, which corresponds to the "frozen" density interaction energy approximation. $E_{B L W}$ is the energy obtained by Ensemble BLW.

\section{Results and Discussion}

As a first application of Ensemble BLW-EDA to metal surfaces, we compare the adsorption of $\mathrm{H}_{2} \mathrm{O}$ and $\mathrm{H}_{2} \mathrm{~S}$ on $\mathrm{Pt}(111)$. As shown Figure 1 for both molecules the most favorable adsorption configuration is atop, with the molecular plane approximately parallel to the surface. The total interaction energy as a function of the $\mathrm{Pt}-\mathrm{O}$ or $\mathrm{Pt}-\mathrm{S}$ distance shows that despite the nominally larger non-covalent radius of sulfur with respect to oxygen (1.8 and $1.5 \AA$ according to Bondi $\left.{ }^{(65}\right)$, the minimum is found around $2.4 \AA$ in both cases. This can be rationalized by the overall stronger interaction of $\mathrm{Pt}-\mathrm{H}_{2} \mathrm{~S}\left(29 \mathrm{kcal} \mathrm{mol}^{-1}\right)$ compared to $\mathrm{Pt}-\mathrm{H}_{2} \mathrm{O}\left(9 \mathrm{kcal} \mathrm{mol}^{-1}\right)$, which allows $\mathrm{S}$ to approach the surface more closely than $\mathrm{O}$ with respect to its size. The BSSE contribution to the interaction energy is similar for $\mathrm{H}_{2} \mathrm{O}$ and $\mathrm{H}_{2} \mathrm{~S}$ (roughly $10 \%$ of the total interaction energy). Therefore, the comparison between the two 

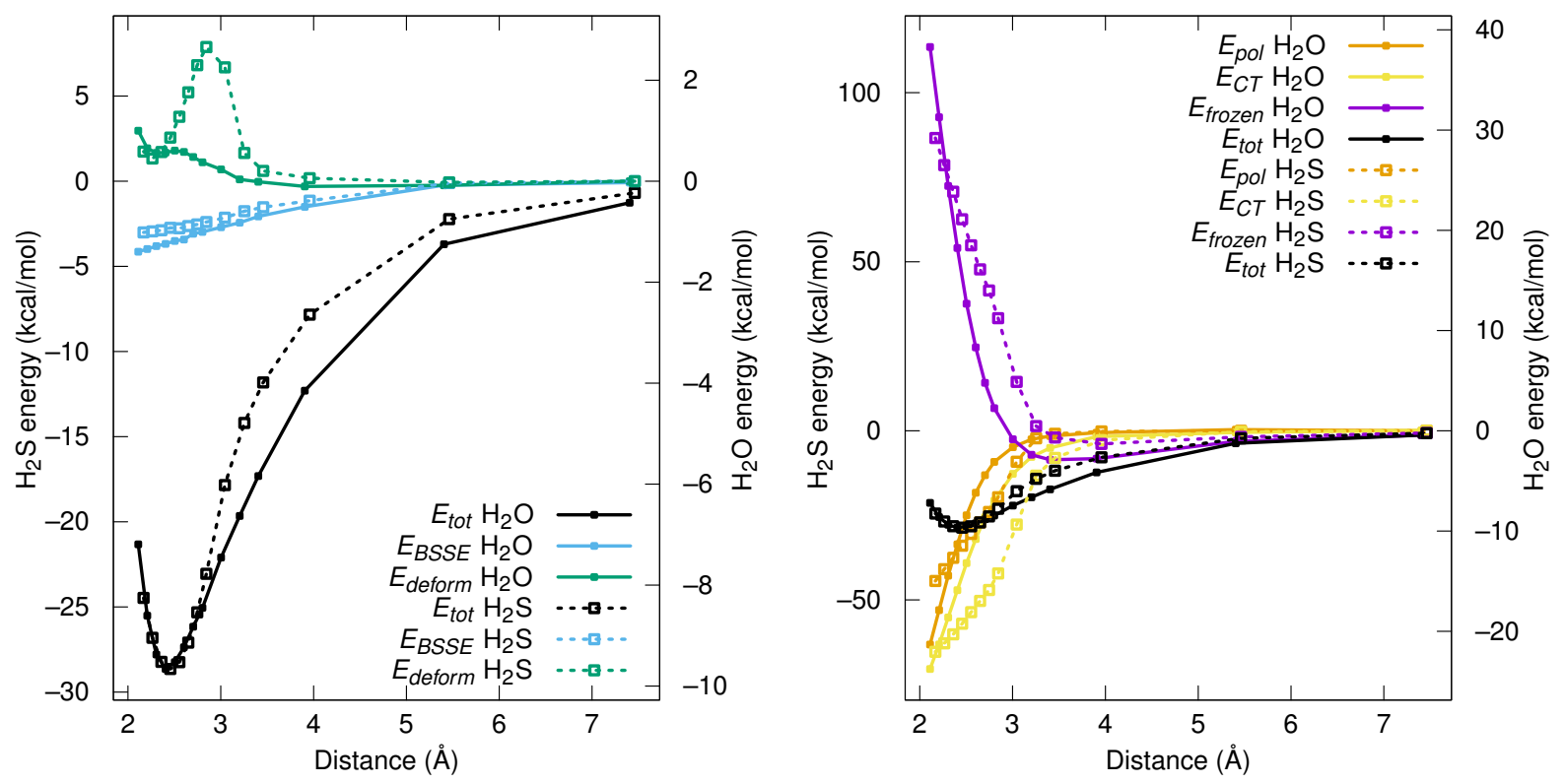

Figure 2: Energy decomposition analysis for $\mathrm{H}_{2} \mathrm{O}$ (full lines, right y axis) and $\mathrm{H}_{2} \mathrm{~S}$ (broken lines, left y-axis). The total interaction energy is compared to the BSSE and the deformation energy on the left, while the right quantifies the frozen monomers, polarization and charge transfer interaction energy. Note that the y-scales are aligned in such a way that the equilibrium interaction energy and the zero interaction energy are aligned for both systems, see SI for separated Figures. 
systems could be performed with a $10 \%$ accuracy without the BSSE correction, for instance for molecular dynamics simulations of $\mathrm{H}_{2} \mathrm{~S}$ dissolved in water. The deformation energy of the two systems show different patterns. While the energetic cost to deform water/Pt(111) to its optimal geometry is very small (at most $0.5 \mathrm{kcal} \mathrm{mol}^{-1}$ ), $\mathrm{H}_{2} \mathrm{~S} / \mathrm{Pt}(111)$ undergoes a deformation up to $7 \mathrm{kcal} \mathrm{mol}^{-1}$ at a distance that is only slightly longer than the equilibrium distance, while it drops at shorter distances to about $1 \mathrm{kcal} \mathrm{mol}^{-1}$. Analyzing the origin of the deformation energy, we identify the deformation of the metal surface as the major contributor. As shown in Figure S2, the Pt atom on which the adsorbate is adsorbed, is "pulled out" of the surface at intermediate distances. After a certain elongation, it goes back to the original position, almost as if the spring had been overstretched and thus broken. Moving to the interaction energy components of the fragments after paying the preparation (deformation) energy (right hand graph of Figure 2), we first observe that the "frozen" term, i.e., the energy cost (or gain) of putting the fragments together without electronic density changes, follows the expected increase in atomic size when replacing oxygen by sulfur: The energy of assembly becomes positive for distances below 2.8 and $3.2 \AA$ for $\mathrm{H}_{2} \mathrm{O}$ and $\mathrm{H}_{2} \mathrm{~S}$, respectively. At larger distances, the interaction energy is already negative without any further electronic relaxation. This is due to the subtle balance between Pauli repulsion and attraction by dispersion interactions. Note, that electrostatic interactions are also included at this stage, but since the isolated metal surfaces do not have significant electrostatic moments (dipole, quadrupole etc.), it barely contributes in the present systems. Allowing the electron density to relax in the presence of the other fragment, but prohibiting any charge transfer (or direct orbital interaction), affords the stabilizing polarization energy $E_{p o l} . \mathrm{H}_{2} \mathrm{~S}$ has a 2.7 times larger polarizability than $\mathrm{H}_{2} \mathrm{O}$ (26.7 and 9.8 a.u. ${ }^{3}$, respectively, $\left.{ }^{66}\right)$. Indeed, there is roughly a factor of three between $E_{p o l}$ for $\mathrm{H}_{2} \mathrm{~S}$ compared to $\mathrm{H}_{2} \mathrm{O}$ at all relevant distances $(>2.3 \AA$ ), in close agreement with the corresponding factor for the total interaction energy. The charge transfer between the metal surface and the adsorbate, is, however, much more important for $\mathrm{H}_{2} \mathrm{~S}$ than for $\mathrm{H}_{2} \mathrm{O}$. This contrasting energy decomposition can be rationalized recalling 
that sulfides are softer bases than oxides and that platinum is a soft acid. Furthermore, interactions with sulfur can benefit from stabilization through hypervalence by increasing the ionic character of bonds. .67

As a conclusion, the stabilizing interaction of a single water molecule with $\mathrm{Pt}(111)$ is due to equal amounts to polarization and to charge transfer. In other words, even in this case of rather weak physisorption, the use of a polarizable force field is unlikely to be enough to capture the physics of the interaction energy accurately. However, a good approximation can be obtained since the two components are well correlated, i.e., counting the polarization energy twice, BLW could capture a reasonable approximation of the total interaction energy. This insight might help the development of more accurate $\mathrm{Pt} / \mathrm{H}_{2} \mathrm{O}$ force fields. $\stackrel{68 \mid 69}{6 h e}$ situation for $\mathrm{H}_{2} \mathrm{~S}$ is more challenging to approximate: charge-transfer dominates the interaction energy, which also induces significant deformations of the surface. Hence, already for $\mathrm{H}_{2} \mathrm{~S}$ adsorption, explicit terms to mimic orbital/charge-transfer interactions are required. The situation can be simplified by excluding surface deformation. Given the scarcity of accurate force fields for the deformation of metal surfaces, this is the preferred setup anyway. Figure S3 shows that on an ideal surface the deformation energy is negligible and the charge-transfer and polarization energies are now as smooth as for water. Furthermore, on an ideal surface, a very similar relative importance of the various contributions is obtained for $\mathrm{H}_{2} \mathrm{O}$ and $\mathrm{H}_{2} \mathrm{~S}$. Since $E_{t o t}$ is not very different when using an ideal $\mathrm{Pt}(111)$ surface, $\mathrm{H}_{2} \mathrm{~S}$ adsorption can be described without taking into account the surface deformation with only a small loss in accuracy. Given that $E_{C T}$ is most strongly affected, this analysis also identifies the charge-transfer as the origin of the deformation.

On a more technical level, we have also investigated the influence of the electronic smearing temperature on the results. In Figure S4 we show that increasing the electronic temperature from $300 \mathrm{~K}$ to $1000 \mathrm{~K}$ does not visibly affect the results. Even at $2000 \mathrm{~K}$ only changes of 3-4 kcal mol-1 are observed, leaving the relative importance of different terms unchanged. As detailed in the SI, the small changes observed can be rationalized keeping in mind that 
higher electronic temperatures lead to the occupation of orbitals lying above the Fermi level.
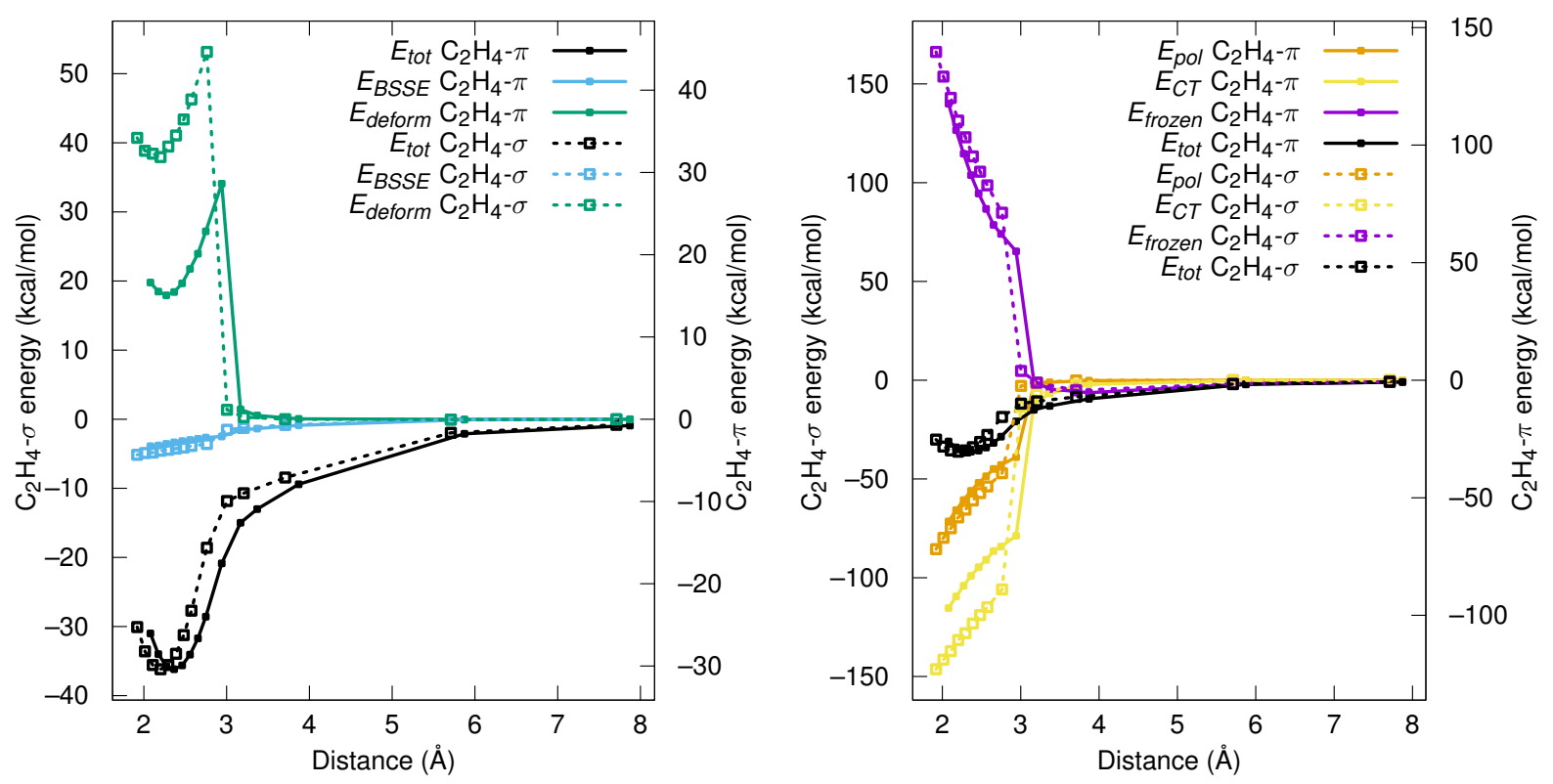

Figure 3: Energy decomposition analysis for di- $\sigma$ (broken lines, left y-axis) and $\pi$ (full lines, right y axis) $\mathrm{C}_{2} \mathrm{H}_{4}$. The total interaction energy is compared to the BSSE and the deformation energy on the left, while the right quantifies the frozen monomers, polarization and charge transfer interaction energy.

The deformation energy observed for $\mathrm{H}_{2} \mathrm{~S}$ is small compared to the one obtained for ethylene adsorption (see Figure 3). This does not come as a surprise, given that the adsorbed geometry (Figure 1) suggests a re-hybridization of the $\mathrm{sp}^{2}$ carbon atoms. This significant geometric rearrangement is best associated with a "state crossing": $\mathrm{sp}^{2}$ at long distances but $\mathrm{sp}^{3}$ at short distances. This state-crossing is non-continuous, as best seen in the deformation energy, although other interaction energy components show a similar discontinuity. As mentioned above, the deformation is mainly due to the deformation of the metal surface, where the Pt atom is partially lifted out of the surface (see Figure S2). The BSSE is again in the order of $10 \%$ of the total interaction energy around the minimum and the total interaction energy is, with 30 and $37 \mathrm{kcal} \mathrm{mol}^{-1}$ for $\pi$ and di- $\sigma$, respectively, similar. Furthermore, the larger geometric deformation (H-C-C-H dihedral angles of 132 and $156^{\circ}$ ) for the di- $\sigma$ mode compared to the $\pi$ mode is nicely captured by the deformation energy. Similar to $\mathrm{H}_{2} \mathrm{~S}$, the maximum deformation energy is obtained when the molecule is close enough to the surface 
to actually "feel" it, but not close enough to form strong covalent bonds. The deformation and the total interaction energy reflect quite well that the $\pi$ mode approaches the surface less closely than the di- $\sigma$ mode (Pt- $\mathrm{C}$ distance of $2.20 \AA$ in $\pi$ and $2.13 \AA$ in di- $\sigma$ ). The right hand side of the graph provides a rationalization: the Pauli repulsion (contained in $E_{\text {frozen }}$ ) is longer-ranged for the $\pi$ bond at the top of a Pt atom, than between the bridge site and carbon atoms that are not strictly on top of Pt atoms. The same observation also applies to the polarization and charge-transfer energy. However, the relative importance of repulsion, polarization and charge-transfer is surprisingly similar for both modes, demonstrating that the nature of the bonding interaction is the same. Nevertheless, the di- $\sigma$ mode maximizes this bonding type which is characterized by $\sim 40 \%$ polarization energy and $\sim 60 \%$ charge-transfer, very similar to the interaction of $\mathrm{H}_{2} \mathrm{~S}$ with the same surface.
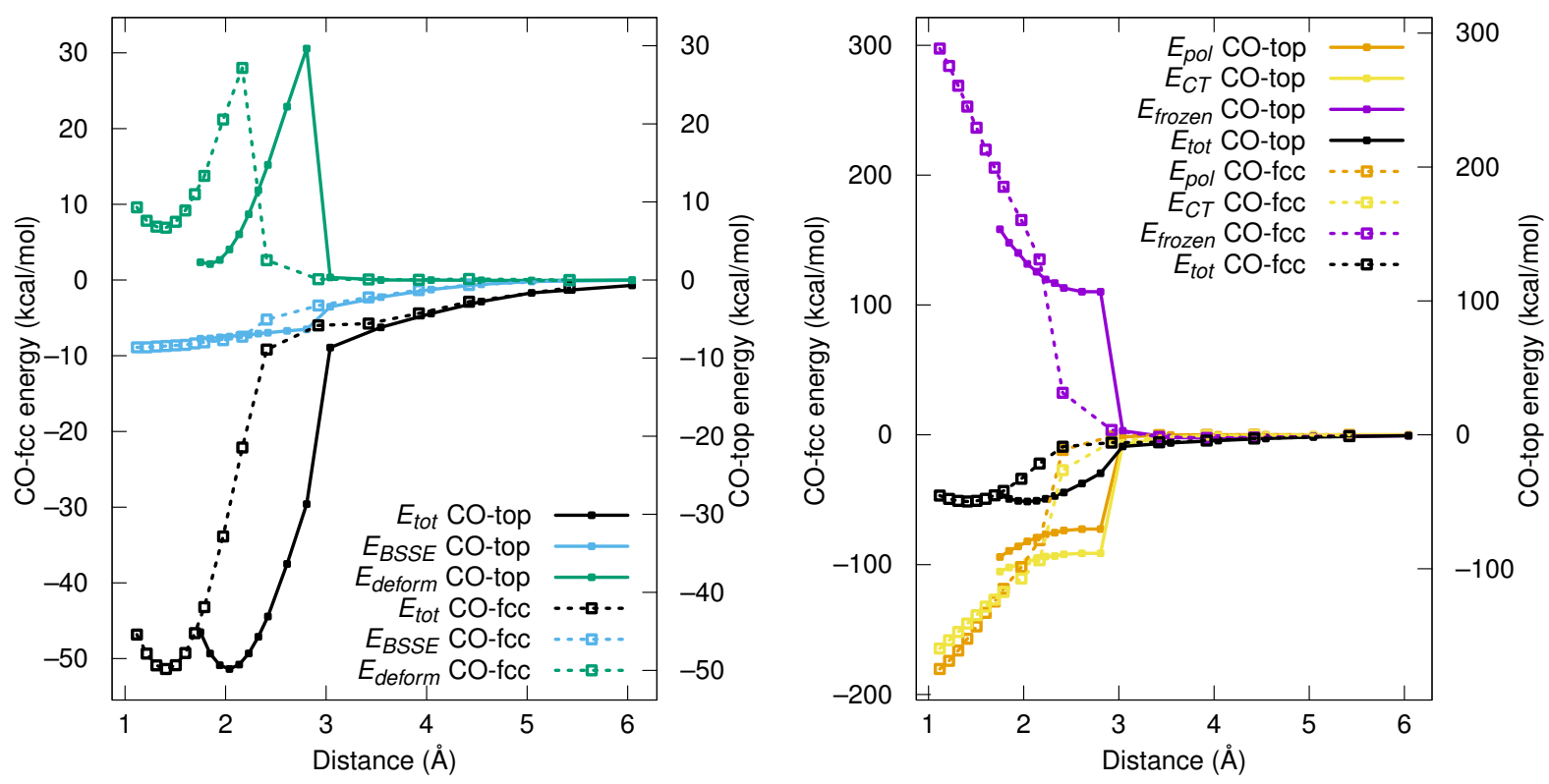

Figure 4: Energy decomposition analysis for top (full lines, right y axis) and fcc (broken lines, left $\mathrm{y}$-axis) $\mathrm{CO}$ on $\mathrm{Pt}(111)$. The total interaction energy is compared to the BSSE and the deformation energy on the left, while the right quantifies the frozen monomers, polarization and charge transfer interaction energy.

The last example we are discussing here is the classic case of $\mathrm{CO}$ adsorption on $\mathrm{Pt}(111)$. One of the challenging questions is whether $\mathrm{CO}$ is adsorbed on top or on bridge/hollow sites and how this evolves as a function of the surface charge. ${ }^{70}$ The following analysis investigates 
exclusively the bonding patterns at the respective adsorption sites, without drawing any conclusions, neither on the reliability of the chosen density functional, nor on the actual site preference of $\mathrm{CO}$ on $\mathrm{Pt}(111)$.

In Figure 4 the data for $\mathrm{CO}$ adsorption on top and fcc sites is compared. At our level of theory, the fcc adsorption site is slightly preferred (-52 vs $-49 \mathrm{kcal} \mathrm{mol}^{-1}$ ). However, these graphs show very clearly the contrasting behavior of $\mathrm{CO}$ on these two sites: The top site experiences a significantly stronger Pauli repulsion, resulting in a longer equilibrium distance ( $2 \AA$ compared to $1.5 \AA$ ). The deformation energy, on the other hand, is slightly lower at the equilibrium distance on the top site than on the fcc site, although the barrier for the state-crossing is roughly equal $\left(\sim 30 \mathrm{kcal} \mathrm{mol}^{-1}\right)$, shifted by $0.75 \AA$ to longer distances for the top mode. Regarding the decomposition of the interaction energy we can first note that the Pauli repulsion at long distances is lower for the fcc mode compared to the top adsorption mode, but at shorter distances rises more quickly for the fcc site. Second, in contrast to the case of $\mathrm{C}_{2} \mathrm{H}_{4}$, the ratio of polarization and charge-transfer energy is quite different for the two adsorption modes. Somewhat surprisingly, the fcc mode shows a similar pattern as $\mathrm{H}_{2} \mathrm{O}$ adsorption, with $E_{p o l}$ and $E_{C T}$ being roughly equal over the entire range of distances. In other words, polarization contributes more to the total binding than for the adsorption of $\mathrm{C}_{2} \mathrm{H}_{4}$. While charge-transfer is relatively more important for the top adsorption mode, it is still far from reaching the importance it takes for ethylene adsorption. We suggest that this "lower than expected" importance of charge-transfer for the adsorption of CO on $\mathrm{Pt}(111)$ is due to the intricate donation and back-donation involved in CO adsorption, which can be seen as a particularly strong polarization effect. In other words, it is the polarization which is particularly strong for $\mathrm{CO}$ adsorption, not the charge-transfer that is small: this dative bond is the strongest bond investigated herein and orbital interactions are without any doubt key for its accurate description. However, our analysis demonstrates that the top site is more sensitive to charge-transfer than adsorption on the hollow site. This is in good agreement with previous reports, ${ }^{70}$ but it is the first time that such a difference is clearly traced back to 
charge-transfer and disentangled from polarization effects.

\section{Conclusions}

In this work, we have presented the extension of the BLW formalism to systems that require fractionally occupied orbitals such as metals at finite temperature. Since the computational cost of the rigorous combinatorial formulation is unpractical, our extension relies on a mean-field approximation to ensemble BLW in the context of mixed-state theory. This approximation is based on a new concept: selfish orbitals. Selfish orbitals are normalized, but their interactions with other orbitals are scaled down according to their occupation number. This mean-field approximation is exact in the case of infinitesimal smearing (i.e. BLW with a gap) and/or 1-block systems (i.e. ensemble KS-DFT). Furthermore, in numerical examples studied, the estimated error in the polarization energy is roughly $0.5 \mathrm{kcal} \mathrm{mol}^{-1}$.

The method has been implemented in $\mathrm{CP} 2 \mathrm{~K}$ and numerical applications have shown that $\mathrm{H}_{2} \mathrm{~S}$ is much more strongly bound to $\mathrm{Pt}(111)$ due to charge-transfer, while the contribution of polarization is on a similar level as for $\mathrm{H}_{2} \mathrm{O}$. The chemisorption of ethylene on $\mathrm{Pt}(111)$ is, however, dominated by the charge-transfer and the two modes (di- $\sigma$ and $\pi$ ) show the same type of bonding, with proportions of different interaction energy components close to the ones of $\mathrm{H}_{2} \mathrm{~S}$. CO adsorption, on the other hand, is shown to depend significantly on the adsorption site: although the bond is five times stronger than for $\mathrm{H}_{2} \mathrm{O}$, the hollow-site adsorption is characterized by a roughly equal contribution of polarization and charge-transfer, just like water adsorption. On the top site charge-transfer is somewhat more important, but

even in this case its importance is relatively smaller than for $\mathrm{H}_{2} \mathrm{~S}$ or $\mathrm{C}_{2} \mathrm{H}_{4}$. We ascribe this particularity to the donation/back-donation of $\mathrm{CO}$, which can be seen as a particularly strong polarization contribution.

As demonstrated herein, BLW-EDA provides a convenient (i.e. easy to use) tool, providing polarization and charge-transfer energies at the metal interface, which is expected to provide 
valuable insight for catalysis and the understanding of the metal/gas and metal/liquid interface.

\section{Acknowledgement}

The authors thank J. Hutter for insightful discussions. Computational resources were generously provided by the mesocenter PSMN. This work was also granted access to the HPC resources of CINES and IDRIS under the allocation 2014-080609 made by GENCI. The PHC Germaine de Staël program "Reactivity at the solid/liquid interface: Better simulation for a better comprehension", number 30617PA, is acknowledged for supporting the collaboration between SNS and MI.

\section{Supporting Information Available}

Additional figures and details on the error estimates are provided in the SI. Furthermore, tables with the numerical data for all Figures are available. Furthermore, a comparison between BSSE corrected total interaction energies and interaction energies using a plane wave basis set is presented.

The following files are available free of charge.

- Geometries.zip: Archive with optimized geometries and their opt-PBE-vdW energies as obtained by VASP.

This material is available free of charge via the Internet at http://pubs.acs.org/.

\section{References}

(1) Heitler, W.; London, F. Wechselwirkung neutraler Atome und homo-polare Bindung nach der Quantenmechanik. Z. Phys. A 1927, 44, 455-472. 
(2) Ruedenberg, K. The Physical Nature of the Chemical Bond. Rev. Mod. Phys. 1962, 34, $326-376$.

(3) Bader, R. F. W. Atoms in Molecules: A Quantum Theory. 1990,

(4) Reed, A. E.; Curtiss, L. A.; Weinhold, F. Intermolecular interactions from a natural bond orbital, donor-acceptor viewpoint. Chem. Rev. 1988, 88, 899-926.

(5) Gonthier, J. F.; Steinmann, S. N.; Wodrich, M. D.; Corminboeuf, C. Quantification of "fuzzy" chemical concepts: a computational perspective. Chem. Soc. Rev. 2012, 41, $4671-4687$.

(6) Tsipis, A. C. DFT challenge of intermetallic interactions: From metallophilicity and metallaromaticity to sextuple bonding. Coord. Chem. Rev. 2017, 345, 229-262.

(7) Pastorczak, E.; Corminboeuf, C. Perspective: Found in translation: Quantum chemical tools for grasping non-covalent interactions. J. Chem. Phys. 2017, 146, 120901.

(8) Philipsen, P. H. T.; Baerends, E. J. Role of the Fermi Surface in Adsorbate-Metal Interactions: An Energy Decomposition Analysis. J. Phys. Chem. B 2006, 110, 1247012479.

(9) Cruz Hernandez, N.; Zicovich-Wilson, C. M.; Fdez. Sanz, J. The constrained space orbital variation analysis for periodic ab initio calculations. J. Chem. Phys. 2006, 124, 194105.

(10) Raupach, M.; Tonner, R. A periodic energy decomposition analysis method for the investigation of chemical bonding in extended systems. J. Chem. Phys. 2015, 142, 194105.

(11) Xin, H.; Linic, S. Analyzing relationships between surface perturbations and local chemical reactivity of metal sites: Alkali promotion of $\mathrm{O} 2$ dissociation on $\mathrm{Ag}(111) . J$. Chem. Phys. 2016, 144, 234704. 
(12) Pecher, J.; Mette, G.; Durr, M.; Tonner, R. Site-Specific Reactivity of Ethylene at Distorted Dangling-Bond Configurations on Si(001). ChemPhysChem 2017, 18, 357-365.

(13) Pecher, J.; Schober, C.; Tonner, R. Chemisorption of a Strained but Flexible Molecule: Cyclooctyne on $\mathrm{Si}(001)$. Chem.-Eur. J. 2017, 23, 5459-5466.

(14) Mo, Y.; Song, L.; Wu, W.; Zhang, Q. Charge transfer in the electron donor-acceptor complex BH3NH3. J. Am. Chem. Soc. 2004, 126, 3974-3982.

(15) Khaliullin, R. Z.; Head-Gordon, M.; Bell, A. T. An efficient self-consistent field method for large systems of weakly interacting components. J. Chem. Phys. 2006, 124, 204105.

(16) Kaduk, B.; Kowalczyk, T.; Van Voorhis, T. Constrained Density Functional Theory. Chem. Rev. 2012, 112, 321-370.

(17) Plaisance, C. P.; van Santen, R. A.; Reuter, K. Constrained-Orbital Density Functional Theory. Computational Method and Applications to Surface Chemical Processes. J. Chem. Theory Comput. 2017, 13, 3561-3574.

(18) Mao, Y.; Ge, Q.; Horn, P. R.; Head-Gordon, M. On the Computational Characterization of Charge-Transfer Effects in Noncovalently Bound Molecular Complexes. J. Chem. Theory Comput. 2018, 14, 2401-2417.

(19) Stoll, H.; Wagenblast, G.; Preuss, H. On the use of local basis sets for localized molecular orbitals. Theor. Chim. Acta 1980, 57, 169-178.

(20) Gianinetti, E.; Raimondi, M.; Tornaghi, E. Modification of the Roothaan equations to exclude BSSE from molecular interaction calculations. Int. J. Quantum Chem. 1996, $60,157-166$.

(21) Nagata, T.; Takahashi, O.; Saito, K.; Iwata, S. Basis set superposition error free selfconsistent field method for molecular interaction in multi-component systems: Projection operator formalism. J. Chem. Phys. 2001, 115, 3553-3560. 
(22) Mo, Y.; Gao, J.; Peyerimhoff, S. D. Energy decomposition analysis of intermolecular interactions using a block-localized wave function approach. J. Chem. Phys. 2000, 112, $5530-5538$.

(23) Khaliullin, R. Z.; Cobar, E. A.; Lochan, R. C.; Bell, A. T.; Head-Gordon, M. Unravelling the Origin of Intermolecular Interactions Using Absolutely Localized Molecular Orbitals. J. Phys. Chem. A 2007, 111, 8753-8765.

(24) Khaliullin, R. Z.; Bell, A. T.; Head-Gordon, M. Analysis of charge transfer effects in molecular complexes based on absolutely localized molecular orbitals. J. Chem. Phys. 2008, 128, 184112.

(25) Azar, R. J.; Head-Gordon, M. An energy decomposition analysis for intermolecular interactions from an absolutely localized molecular orbital reference at the coupledcluster singles and doubles level. J. Chem. Phys. 2012, 136, 024103.

(26) Thirman, J.; Head-Gordon, M. An energy decomposition analysis for second-order Moller-Plesset perturbation theory based on absolutely localized molecular orbitals. J. Chem. Phys. 2015, 143, 084124.

(27) Smits, G. F.; Krol, M. C.; Hart, W. J. v. d.; Altona, C. Theoretical investigations of the nature of intramolecular interactions. Mol. Phys. 1986, 59, 209-225.

(28) Mo, Y.; Wu, W.; Song, L.; Lin, M.; Zhang, Q.; Gao, J. The Magnitude of Hyperconjugation in Ethane: A Perspective from Ab Initio Valence Bond Theory. Angew. Chem., Int. Ed. 2004, 43, 1986-1990.

(29) Wodrich, M. D.; Gonthier, J. F.; Steinmann, S. N.; Corminboeuf, C. How Strained are Carbomeric-Cycloalkanes? J. Phys. Chem. A 2010, 114, 6705-6712.

(30) Levine, D. S.; Horn, P. R.; Mao, Y.; Head-Gordon, M. Variational Energy Decomposition 
Analysis of Chemical Bonding. 1. Spin-Pure Analysis of Single Bonds. J. Chem. Theory Comput. 2016, 12, 4812-4820.

(31) Levine, D. S.; Head-Gordon, M. Energy decomposition analysis of single bonds within Kohn-Sham density functional theory. Proc. Natl. Acad. Sci. 2017, 114, 12649-12656.

(32) Couty, M.; Bayse, C. A.; Hall, M. B. Extremely localized molecular orbitals (ELMO): a non-orthogonal Hartree-Fock method. Theor. Chem. Acc. 1997, 97, 96-109.

(33) Fornili, A.; Sironi, M.; Raimondi, M. Determination of extremely localized molecular orbitals and their application to quantum mechanics/molecular mechanics methods and to the study of intramolecular hydrogen bonding. J. Mol. Struct. 2003, 632, 157-172.

(34) Genoni, A.; Ghitti, M.; Pieraccini, S.; Sironi, M. A novel extremely localized molecular orbitals based technique for the one-electron density matrix computation. Chem. Phys. Lett. 2005, 415, 256-260.

(35) Fornili, A.; Moreau, Y.; Sironi, M.; Assfeld, X. On the suitability of strictly localized orbitals for hybrid QM/MM calculations. J. Comput. Chem. 2006, 27, 515-523.

(36) Genoni, A. X-ray Constrained Extremely Localized Molecular Orbitals: Theory and Critical Assessment of the New Technique. J. Chem. Theory Comput. 2013, 9, 30043019.

(37) Steinmann, S. N.; Jana, D. F.; Wu, J. I.-C.; Schleyer, P. v. R.; Mo, Y.; Corminboeuf, C. Direct Assessment of Electron Delocalization Using NMR Chemical Shifts. Angew. Chem., Int. Ed. 2009, 48, 9828-9833.

(38) Elgabarty, H.; Khaliullin, R. Z.; Kuhne, T. D. Covalency of hydrogen bonds in liquid water can be probed by proton nuclear magnetic resonance experiments. Nat. Commun. 2015, 6 . 
(39) Steinmann, S. N.; Vogel, P.; Mo, Y.; Corminboeuf, C. The norbornene mystery revealed. Chem. Commun. 2011, 47, 227-229.

(40) Steinmann, S. N.; Mo, Y.; Corminboeuf, C. How do electron localization functions describe $\pi$-electron delocalization? Phys. Chem. Chem. Phys. 2011, 13, 20584-20592.

(41) Bomble, L.; Steinmann, S. N.; Perez-Peralta, N.; Merino, G.; Corminboeuf, C. Bonding analysis of planar hypercoordinate atoms via the generalized BLW-LOL. J. Comput. Chem. 2013, 34, 2242-2248.

(42) Mo, Y.; Gao, J. Polarization and Charge-Transfer Effects in Aqueous Solution via Ab Initio QM/MM Simulations. J. Phys. Chem. B 2006, 110, 2976-2980.

(43) Giese, T. J.; Chen, H.; Huang, M.; York, D. M. Parametrization of an Orbital-Based Linear-Scaling Quantum Force Field for Noncovalent Interactions. J. Chem. Theory Comput. 2014, 10, 1086-1098.

(44) Demerdash, O.; Mao, Y.; Liu, T.; Head-Gordon, M.; Head-Gordon, T. Assessing manybody contributions to intermolecular interactions of the AMOEBA force field using energy decomposition analysis of electronic structure calculations. J. Chem. Phys. 2017, $147,161721$.

(45) Bambagioni, V.; Bevilacqua, M.; Bianchini, C.; Filippi, J.; Lavacchi, A.; Marchionni, A.; Vizza, F.; Shen, P. K. Cover Picture: Self-Sustainable Production of Hydrogen, Chemicals, and Energy from Renewable Alcohols by Electrocatalysis (ChemSusChem 7/2010). ChemSusChem 2010, 3, 765-765.

(46) Liu, P.; Norskov, J. K. Ligand and ensemble effects in adsorption on alloy surfaces. Phys. Chem. Chem. Phys. 2001, 3, 3814-3818.

(47) Gianinetti, E.; Vandoni, I.; Famulari, A.; Raimondi, M. Extension of the SCF-MI 
method to the case of $\mathrm{K}$ fragments one of which is an open-shell system. Adv. Quantum Chem. 1999, 31, 251-266.

(48) Horn, P. R.; Sundstrom, E. J.; Baker, T. A.; Head-Gordon, M. Unrestricted absolutely localized molecular orbitals for energy decomposition analysis: Theory and applications to intermolecular interactions involving radicals. J. Chem. Phys. 2013, 138, 134119.

(49) Yang, W.; Mori-Sanchez, P.; Cohen, A. J. Extension of many-body theory and approximate density functionals to fractional charges and fractional spins. J. Chem. Phys. 2013, 139, 104114.

(50) Steinmann, S. N.; Yang, W. Wave function methods for fractional electrons. J. Chem. Phys. 2013, 139, 074107.

(51) Khaliullin, R. Z.; VandeVondele, J.; Hutter, J. Efficient Linear-Scaling Density Functional Theory for Molecular Systems. J. Chem. Theory Comput. 2013, 9, 4421-4427.

(52) Mayer, I.; Surjan, P. R. Localization and delocalization. II. Role of overlap in interbond interactions. J. Chem. Phys. 1984, 80, 5649-5658.

(53) CP2K Open Source Molecular Dynamics, available at https://www.cp2k.org/, https: //wWW.cp2k.org/.

(54) Kresse, G.; Hafner, J. Ab initio molecular dynamics for liquid metals. Phys. Rev. B 1993, 47, 558.

(55) Kresse, G.; Furthmuller, J. Efficient iterative schemes for ab initio total-energy calculations using a plane-wave basis set. Phys. Rev. B 1996, 54, 11169.

(56) Klimes, J.; Bowler, D. R.; Michaelides, A. Chemical accuracy for the van der Waals density functional. J. Phys.: Condens. Matter 2010, 22, 022201.

(57) Steinmann, S. N.; Corminboeuf, C. Comprehensive Benchmarking of a DensityDependent Dispersion Correction. J. Chem. Theory Comput. 2011, 7, 3567-3577. 
(58) Gautier, S.; Steinmann, S. N.; Michel, C.; Fleurat-Lessard, P.; Sautet, P. Molecular adsorption at $\mathrm{Pt}(111)$. How accurate are DFT functionals? Phys. Chem. Chem. Phys. 2015, 17, 28921-28930.

(59) Blochl, P. E. Projector augmented-wave method. Phys. Rev. B 1994, 50, 17953.

(60) Kresse, G.; Joubert, D. From ultrasoft pseudopotentials to the projector augmented-wave method. Phys. Rev. B 1999, 59, 1758.

(61) VandeVondele, J.; Hutter, J. Gaussian basis sets for accurate calculations on molecular systems in gas and condensed phases. J. Chem. Phys. 2007, 127, 114105.

(62) Goedecker, S.; Teter, M.; Hutter, J. Separable dual-space Gaussian pseudopotentials. Phys. Rev. B 1996, 54, 1703-1710.

(63) Boys, S. F.; Bernardi, F. The calculation of small molecular interactions by the differences of separate total energies. Some procedures with reduced errors. Mol. Phys. 1970, 19, $553-566$.

(64) Steinmann, S. N.; Corminboeuf, C.; Wu, W.; Mo, Y. Dispersion-Corrected Energy Decomposition Analysis for Intermolecular Interactions Based on the BLW and dDXDM Methods. J. Phys. Chem. A 2011, 115, 5467-5477.

(65) Bondi, A. van der Waals Volumes and Radii. J. Phys. Chem. 1964, 68, 441-451.

(66) Miller, T. M. CRC Handbook of Chemistry and Physics; Taylor \& Francis Group.

(67) Reed, A. E.; Schleyer, P. v. R. Chemical bonding in hypervalent molecules. The dominance of ionic bonding and negative hyperconjugation over d-orbital participation. $J$. Am. Chem. Soc. 1990, 112, 1434-1445.

(68) Steinmann, S. N.; Fleurat-Lessard, P.; Gotz, A. W.; Michel, C.; Ferreira de Morais, R.; Sautet, P. Molecular mechanics models for the image charge, a comment on "Including 
image charge effects in the molecular dynamics simulations of molecules on metal surfaces". J. Comput. Chem. 2017, 38, 2127-2129.

(69) Steinmann, S. N.; Ferreira De Morais, R.; Gotz, A. W.; Fleurat-Lessard, P.; Iannuzzi, M.; Sautet, P.; Michel, C. Force Field for Water over Pt(111): Development, Assessment, and Comparison. J. Chem. Theory Comput. 2018, 14, 3238-3251.

(70) Kalhara Gunasooriya, G. T. K.; Saeys, M. CO Adsorption Site Preference on Platinum: Charge Is the Essence. ACS Catal. 2018, 8, 3770-3774. 


\section{Graphical TOC Entry}

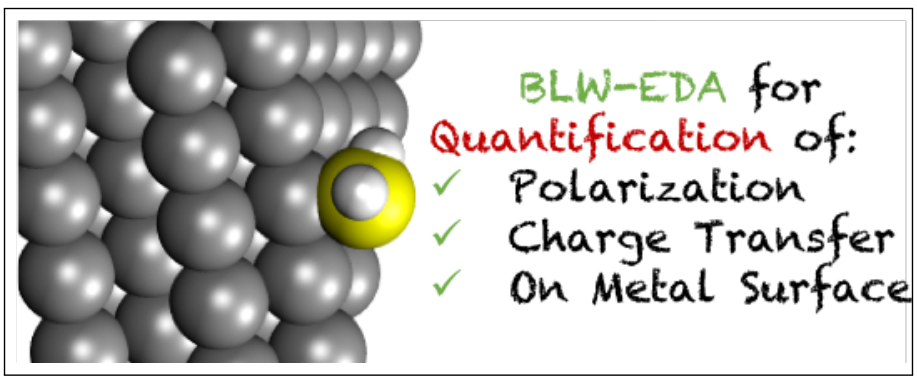

